\title{
Numerical Investigation on the Effects of Structural Parameters of Labyrinth Cavity on Sealing Performance
}

\author{
Jianmei Feng $(\mathbb{D}$, Lingzi Wang, Hang Yang, and Xueyuan Peng \\ School of Energy and Power Engineering, Xian Jiaotong University, Xian 710049, China \\ Correspondence should be addressed to Jianmei Feng; jmfeng@xjtu.edu.cn
}

Received 8 April 2018; Revised 19 June 2018; Accepted 2 July 2018; Published 19 July 2018

Academic Editor: Sandro Longo

Copyright (c) 2018 Jianmei Feng et al. This is an open access article distributed under the Creative Commons Attribution License, which permits unrestricted use, distribution, and reproduction in any medium, provided the original work is properly cited.

\begin{abstract}
This paper presents a study on the leakage characteristics of a labyrinth piston compressor. A computational fluid dynamics model was applied to predict the effects of different structural parameters of the labyrinth seal on the sealing performance. The velocity field through the sealing clearance and labyrinth cavity was demonstrated and analyzed. An experimental rig was built to validate the simulation model, and the results of the simulation and experimental data showed a good agreement. The effects of the cavity width, cavity depth, tooth thickness, and clearance of the seal on the leakage characteristics were discussed in detail. The results showed that, except for the significant influence of the labyrinth clearance, $d / \delta$ (the ratio of cavity depth to labyrinth clearance) should be bigger than 2.7 to achieve the minimum leakage flow, and the effect of the varied cavity width can be ignored. Moreover, when the piston length and cavity width remain constant, the thinner the tooth thickness, the greater the number of labyrinth cavities that can be arranged, and the higher the sealing performance achieved.
\end{abstract}

\section{Introduction}

In the labyrinth piston compressor shown in Figure 1, a noncontact labyrinth seal was used between the piston and cylinder wall, as well as between the piston rod and packing. The annular labyrinth grooves were machined around the piston, piston rod, and cylinder wall, and as indicated in the sectional view, there are many small cavities on the two sides of the tiny labyrinth channel. In the compressor, the internal leakage is the main source of leakage rather than external leakage, and thus the labyrinth structure on the piston has been mostly discussed.

By using a labyrinth seal instead of a piston ring, the reciprocating compressor has many advantages, such as oil-free gas compression and transport, nonlubricated maintenance, and no friction loss. A labyrinth piston compressor can provide environmentally friendly products as high purity gases and can be widely used in various industries [1]. However, the wider application of labyrinth piston compressors has been limited because they show a worse sealing performance compared to traditional compressors, which have piston rings.
Thus, studying the influence of the structural parameters of a labyrinth seal has a great significance in optimizing the sealing performance in labyrinth piston compressors.

As early as in 1908, Martin [2] began studying the mass flow rate in a labyrinth gap, and over the following 50 years, different types of analytical calculation methods of a labyrinth seal flow were explored [3-6]. In the late twentieth century, a series of experimental and numerical studies were carried out to investigate the labyrinth mechanism in rotating machinery [7-9]. Later, many researchers began continuously enriching the labyrinth used in rotating machinery, and by the beginning of the 21st century, valuable research results on the labyrinth seal in rotating machinery began being published [10-14].

Wang et al. [15] simulated the distribution of a static flow field on two kinds of seals, and their results showed that a stepped labyrinth is more efficient than an interlocking seal. Suryanarayanan and Morrison [16-18] studied the relationship between the flow coefficient and transmission coefficient inside the labyrinth channels in detail, and the influence of the tooth type, compression ratio, clearance, and Reynolds 


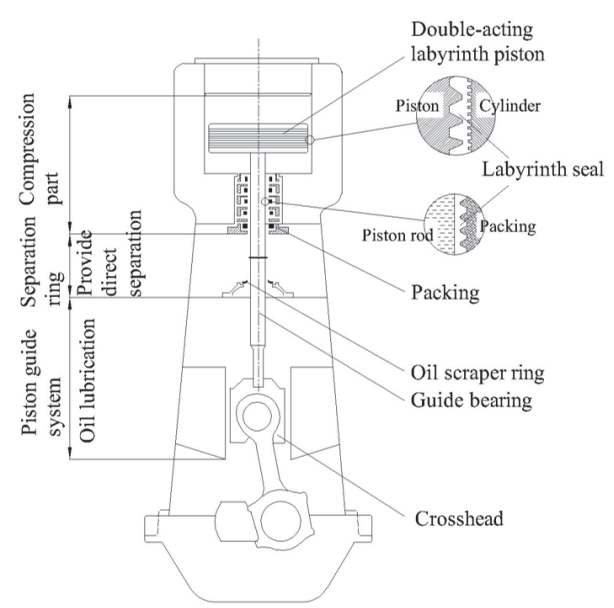

FIgURE 1: A typical labyrinth piston compressor.

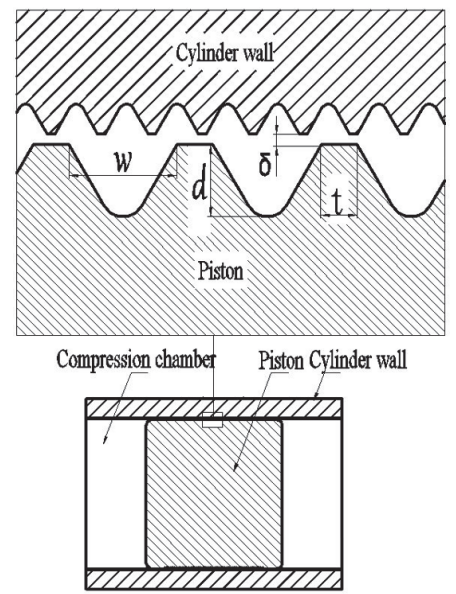

FIgURE 2: Simplified model. number were obtained. Kim and Cha [19] combined the computational fluid dynamics (CFD) method and a theoretical analysis to discuss the influence of the structural arrangement and pressure ratio on the sealing efficiency and found that a stepped labyrinth seal is more efficient when the labyrinth clearance is larger. Bozzi et al. [20] performed a more detailed analysis on the heat transfer in a labyrinth seal. Pugachev et al. [21, 22] used a CFD model to research the direction of the main vortex in a short chamber with a backward-facing step and numerically and experimentally verified that the segmentation negatively affects the leakage performance and stiffness coefficients of the brush-labyrinth sealing configuration. Yuan et al. [23] established a CFD model to optimize the seal design for supercritical carbon dioxide in turbomachinery, and the model was then confirmed based on the experimental results.

The majority of studies on labyrinth seals are concentrated on rotating machinery, whereas very few studies have targeted the labyrinth piston compressors directly. Because the piston only does reciprocating motion and is shaped like a column, many of the conclusions that were drawn from rotating machinery are no longer suitable for labyrinth piston compressors. For example, the stepped labyrinth seal, which is proved to be more efficient than the straight seal, is unsuitable for application in reciprocating compressors.

Effectively controlling the gas leak is a serious and difficult technical problem for the labyrinth piston compressor. To guide the design of a labyrinth seal in the compressors, determining the influence of the structural parameters of a labyrinth on the sealing performance is important. In this study, the effects of various structural parameters, such as the labyrinth clearance, the width and depth of the labyrinth cavity, and the number of labyrinth cavities, were numerically investigated, and the simulation method was verified experimentally.

\section{Simulation Method}

2.1. Geometrical Model. According to Meng and Xia [24], isosceles trapezoid and triangle shaped cavities achieve the best sealing effect compared with circular, rectangular, and right-angle cavities. Considering the convenience of processing in reality, an isosceles trapezoid cavity with a circular bottom was used in this research, and the symbols for different structural parameters are depicted in Figure 2.

To fully understand the flow field distribution in the labyrinth seal, the piston, entire labyrinth seal channel, and two compression chambers were combined as an entire structure to be simulated. The square in the middle is the piston, and the connecting rod is removed to simplify the calculation. In this research, the studied pressure ratios are 3 to 8 . The original diameter of the piston is $150 \mathrm{~mm}$, and the magnitude of $\delta$ is generally on the order of $0.1 \mathrm{~mm}$. The length of the piston was chosen according to the piston stroke and mechanical design handbook. On the side of the piston, there is a slight slope in case of an interaction between the piston and inner wall of the cylinder.

In most cylinders, there are small labyrinth cavities in the inner wall, as shown in Figure 2. Theoretically, small labyrinth cavities can assist the labyrinth cavities on the piston to strengthen the sealing effect. When the labyrinth cavities are on both sides, the kinetic energy of the high-speed airflow will be dissipated more fully through the clearance, and the effect of the labyrinth seal will be better.

2.2. Numerical Approach. The flow rate through the labyrinth seal is the maximum leakage flow rate when the flow field becomes fully stable, and thus a steady-state simulation was carried out. The simulation of the flow field is based on a numerical solution of the basic equations of fluid mechanics, i.e., the continuity equation, namely,

$$
\frac{\partial \rho}{\partial t}+\nabla \cdot(\rho \vec{v})=0
$$

The momentum balance is described as

$$
\frac{\partial(\rho \vec{v})}{\partial t}+\nabla \cdot(\rho \vec{v} \vec{v})=-\nabla p+\nabla \tau+\vec{S}
$$




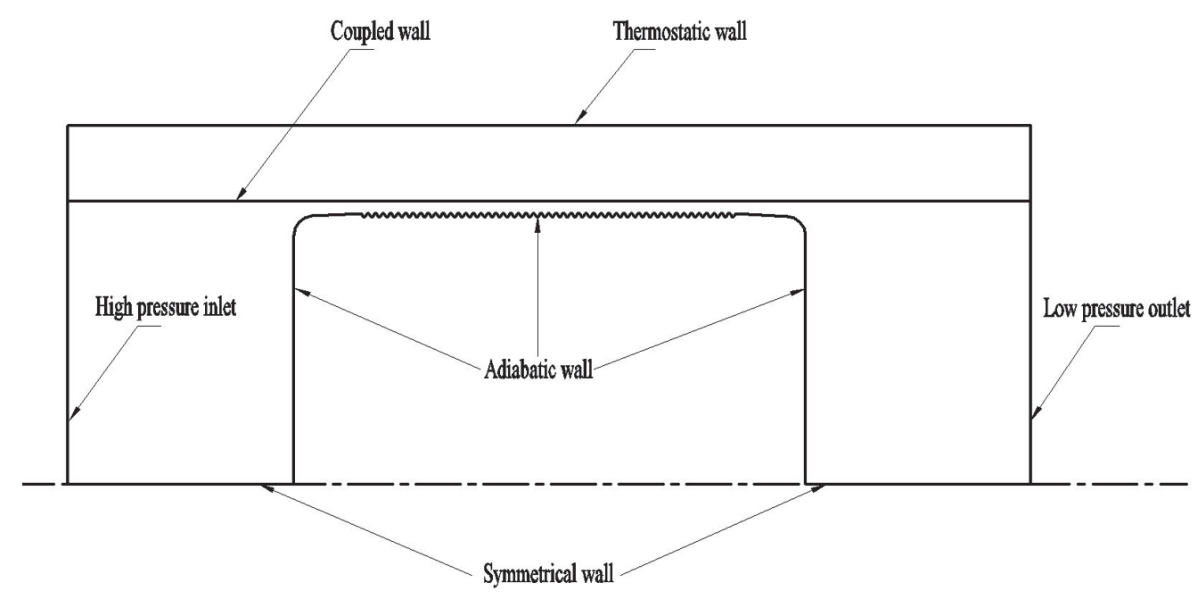

Figure 3: Boundary conditions.

The energy conservation equation is

$$
\frac{\partial(\rho T)}{\partial t}+\nabla(\rho \vec{v} T)=\frac{h}{c_{p}} \Delta T-\nabla q
$$

To consider the compressibility of the gas, the airflow is assumed to comply with the ideal gas law, and thus the state equation is as follows:

$$
p=\rho R T
$$

In the labyrinth channel, when the fluid flows through the tiny clearance to the cavity, a swirl flow will form. In the realizable $k-\varepsilon$ model, the coefficient used in calculating the turbulent viscosity was correlated with the strain rate rather than a constant [25] and thus was approved to be suitable to simulate a swirl flow. In this research, the realizable $k-\varepsilon$ model is used to compute the turbulence transport contribution

The boundary settings are illustrated in Figure 3 . The outlet pressure was continuously set to the ambient pressure, 0.1 $\mathrm{MPa}$; the high inlet pressure varied from 0.3 to $0.8 \mathrm{MPa}$, and thus the pressure ratios were 3 to 8 . The outlet temperature was the ambient temperature, $300 \mathrm{~K}$, and the temperature of the inlet was set to the true temperature discharged from the air source compressor. The cylinder's outer wall made direct contact with the cooling water, which circulated in a timely manner and removed the heat from the cylinder wall instantly. The flow of the water circulation was significant, and the flow rate could be adjusted; thus, the discharge gas temperature was generally 310 to $330 \mathrm{~K}$ when the pressure ratio changed from 3 to 8 , and the temperature of the outer wall of the cylinder was usually kept at $300 \mathrm{~K}$, or almost the same temperature as the cooling water. The turbulent intensity (5\%) and hydraulic diameter (half of the cylinder diameter) were defined at the inlet boundary.

Because the physical model is symmetrical on two sides, the computation model takes the half of region. The velocity is set as zero on the symmetrical wall, and the other physical quantities are completely symmetric. It can be expressed as below:

$$
\phi_{1, J}=\phi_{2, J}
$$

The model equations were discretized with the finite volume method. The pressure-velocity field was obtained by employing the SIMPLE algorithm, and the approximation of state variables is realized by a second-order upwind scheme.

2.3. Grid Independence Verification. As shown in Figure 4, in the labyrinth compressor, the cylinder wall and the compressor chambers are of different orders in terms of size with that of the labyrinth structure. A two-dimensional unstructured grid for the labyrinth and compression chamber was generated. A triangle mesh was used in the labyrinth seal part to fit the labyrinth cavity, which had a triangular shape, whereas in the parts of the cylinder wall and compression chambers, which had rectangular shapes, bigger sized quadrilateral elements were applied.

The difference in scale of the flow field can be presented by setting the mesh scale of the two chambers to 10 times bigger than that of the clearance, and a rapid convergence in the calculation could also be achieved.

Table 1 shows the comparison results of different mesh sizes. The "size" in the first column indicates the grid size of the two compression chambers. When the grid sizes were 1 and $0.5 \mathrm{~mm}$, the mass flow rate of the leakage was much smaller than at $0.1 \mathrm{~mm}$. When the mesh size became larger than $0.2 \mathrm{~mm}$, the number of total elements was only about $25 \%$ of the $0.1 \mathrm{~mm}$ case, whereas the leakage mass flow was only $0.2 \%$ lower. Therefore, considering the simulation time and accuracy, $0.2 \mathrm{~mm}$ was chosen for the mesh size of the compression chamber, whereas for the labyrinth seal, the mesh size was set to $0.02 \mathrm{~mm}$.

\section{Validation Experiment}

The static labyrinth compressor test rig, shown in Figure 5, was built to verify the feasibility of the simulation method. 


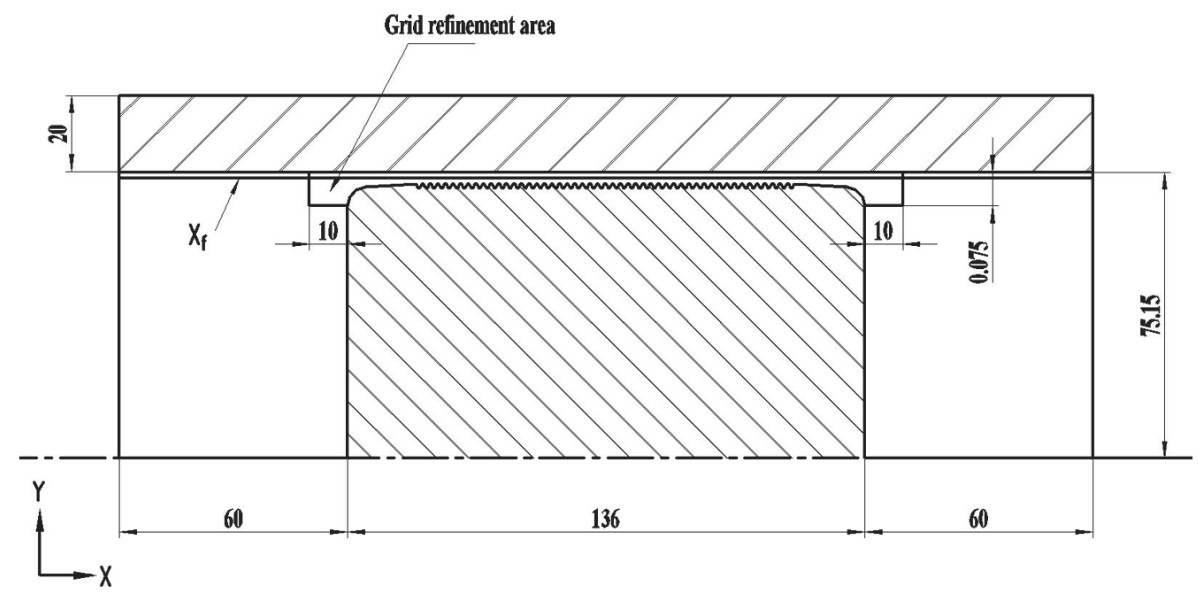

Figure 4: Division of the flow field.

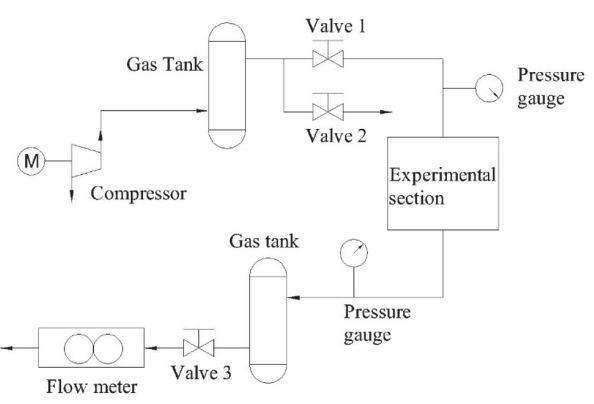

FIgURE 5: Test system.

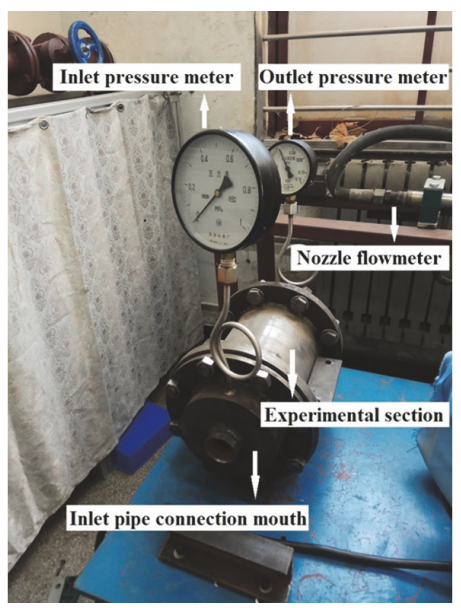

FIgURE 6: Test bench.

First, the air source compressor was started, which would compress the air to about 1.0 MPa. After the air pressure in the storage tank became stable, valve 1 and valve 2 were regulated to adjust the inlet pressure. Figure 6 shows an image of the real experimental section, and Figure 7 shows a sectional view of the test apparatus.

In the experimental section, the gas flowed into the bottom orifice, following the direction of the arrows, entered

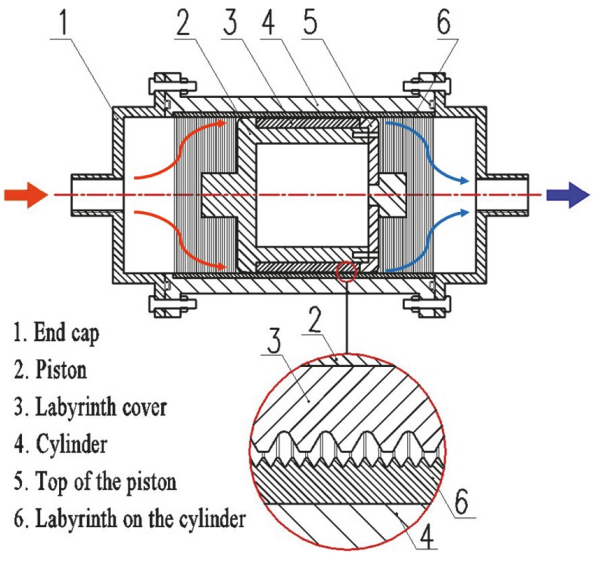

Figure 7: Schematic of labyrinth configuration.

TABLE 1: Mesh independence test.

\begin{tabular}{lcc}
\hline Grid size $/ \mathrm{mm}$ & Total elements & Mass flow of leakage $/ \times 10^{-2} \mathrm{~kg} \cdot \mathrm{s}^{-1}$ \\
\hline 1 & 80,018 & 1.5309 \\
0.5 & 268,760 & 1.5875 \\
0.2 & $1,551,123$ & 1.5983 \\
0.1 & $6,146,194$ & 1.6060 \\
\hline
\end{tabular}

through the labyrinth seals, and then flowed out. When the gas exited from the test section, it went into the gasholder and then flowed through the nozzle flow meter. Finally, the gas was discharged into the environment. When the entire system became stable, the value of the suction temperature, the difference in water column height, the nozzle upstream gas temperature, and the environmental pressure could be measured to calculate the leakage flow rate.

The uncertainty of the test results mainly comes from the systematic errors of the acquisition and measurement equipment. According to the error of the nozzle flow meter, the relative error of the leakage mass flow rate is $2.51 \%$. 


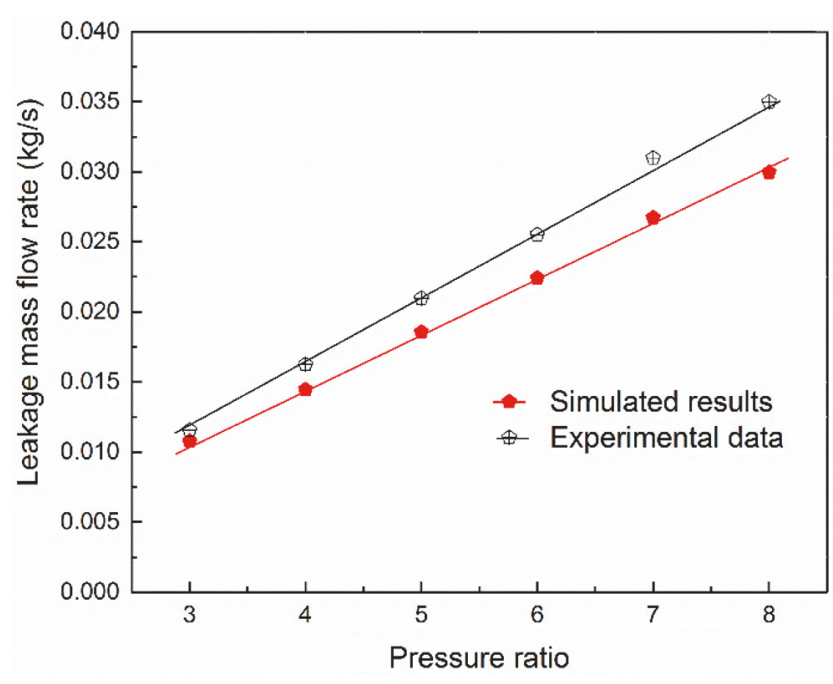

FIGURE 8: Comparison of the experimental data and the simulation results.

\section{Results Discussion}

4.1. Model Validation. Figure 8 indicates that the leakage mass flow rate of the experimental data and the simulated results show a proportional increase with an increase in the pressure ratio when the other conditions are the same; however, the measured leakage mass flow rate was generally higher than the simulated value.

The difference mainly came from the measurement error during the experiments, and the accuracy of the manufactured labyrinth cavities was lower than the ideal value set in the model; thus, the measured leakage was slightly higher. Both the experimental and the simulated results of the labyrinth leakage show a strong linear dependency with an increase in the pressure ratio and have almost the same slope. The relative error between the experimental data and simulated results was less than $10 \%$, and thus the simulation method was shown to be reasonable.

4.2. Distribution of Pressure and Temperature. To reveal the leakage characteristics along the clearance, a horizontal line $X_{f}$ was set in the middle of the labyrinth clearance, as shown in Figure 4 . The pressure and temperature along $X_{f}$ were monitored, the results of which are demonstrated in Figure 9 $(\delta=0.15 \mathrm{~mm}, w=1.5 \mathrm{~mm}, d=1.0 \mathrm{~mm}, t=0.5 \mathrm{~mm})$, when the pressure ratio was 3 . In the labyrinth clearance, the pressure decreased gradually, and from the inlet to the outlet, the pressure increasingly decreased, indicating that the sealing effect of the labyrinth became increasingly significant.

It can also be seen that the temperature oscillates with gradually greater amplitudes from the inlet to the outlet of the labyrinth clearance. This can be attributed to the dramatic increase in speed of the high-pressure fluid going through the tiny labyrinth clearance and the corresponding decrease in the pressure of the fluid. At the same time, its internal energy drops, and the temperature of the fluid rapidly decreases. Subsequently, when the fluid reaches the labyrinth cavity, which has an abruptly increased flow space, a vortex will form owing to the shape of the cavity, as shown in Figures 10(b), $10(\mathrm{c})$, and $10(\mathrm{~d})$, and thus the fluid velocity will suddenly decrease. Because the time of the entire process is too short to include the heat transfer, when the fluid arrives at the next labyrinth cavity, its temperature will recover to the original value. Therefore, in the labyrinth seal, the seal effect becomes increasingly significant; that is, the drop in temperature in the labyrinth seal will increase, and the kinetic energy dissipation function of the labyrinth cavity will be increasingly clearer.

From Figure 10, we can see that, for every labyrinth between two chambers, because the outlet pressure is only 2 bar lower than the inlet pressure, the flow within the entire labyrinth seal is subsonic.

4.3. Comparison of Different Cylinder Walls. The discharge coefficients of compressors having cylinder walls with and without labyrinth cavities, respectively, were compared under different pressure ratios, the results of which are shown in Figure 11. Consistent with the theoretical analysis, the sealing performance of a cylinder with labyrinth cavities was better than that of a smooth cylinder, whose discharge coefficient was about $25 \%$ higher on average.

Because the small labyrinth cavities on a cylinder are difficult to fabricate, many labyrinth compressors only have a labyrinth on the piston, which is also the dominating sealing part. In further research and calculation, only the structures of a labyrinth cavity on the piston were considered, and thus the inner surface of the cylinder was a smooth wall without a labyrinth cavity to simplify the calculation (Figures 3 and 4).

\subsection{Effects of Labyrinth Cavity Parameters}

4.4.1. Discharge Coefficient. The discharge coefficient refers to the ratio of the actual flow to the ideal flow through a specific channel. It is a dimensionless quantity and is often used to describe the sealing efficiency [7, 16-19, 26-29]:

$$
C_{d}=\frac{m_{\text {real }}}{m_{\text {ideal }}} .
$$

In (6), $m_{\text {real }}$ is the real leakage mass flow rate obtained through the experiments or numerical simulation, and $m_{\text {ideal }}$ indicates the theoretical mass flow rate for isentropic conditions:

$$
\begin{gathered}
m_{\text {ideal }}=\rho_{e} v_{e} A_{e}=A_{e}\left(\rho_{0} p_{0}\right)^{1 / 2}\left(2 \frac{k}{k-1}\right)^{1 / 2} \\
\cdot\left[\left(\frac{p_{1}}{p_{0}}\right)^{2 / k}-\left(\frac{p_{1}}{p_{0}}\right)^{(k+1) / k}\right]^{1 / 2} \cdot
\end{gathered}
$$

Here, $A$ is the cross-sectional area at the outlet of the channel; $\rho_{0}$ and $p_{0}$ are the density and static pressure at the inlet of the flow channel, and $p_{e}$ is the static pressure at the outlet of the flow channel; and $k$ is the adiabatic coefficient of the working medium. The smaller the discharge coefficient, the better the labyrinth seal performance. 


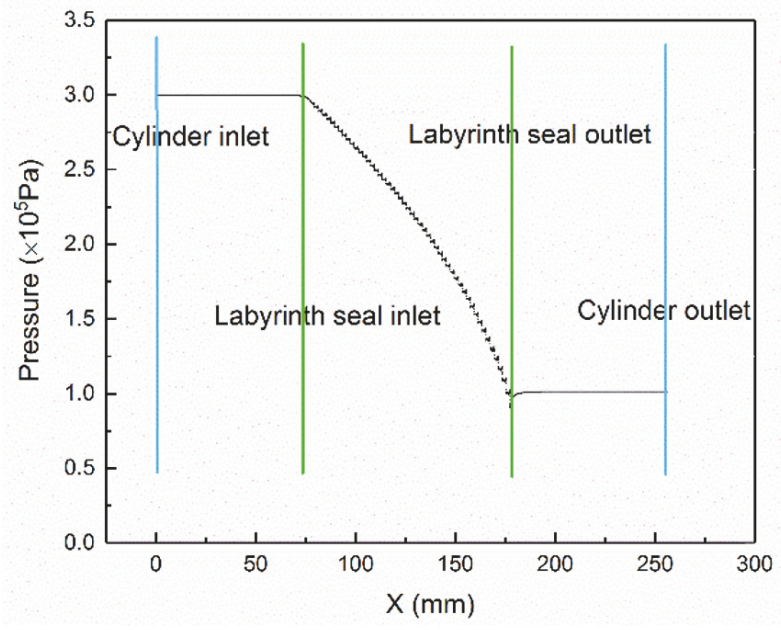

(a) Pressure along $X_{f}$

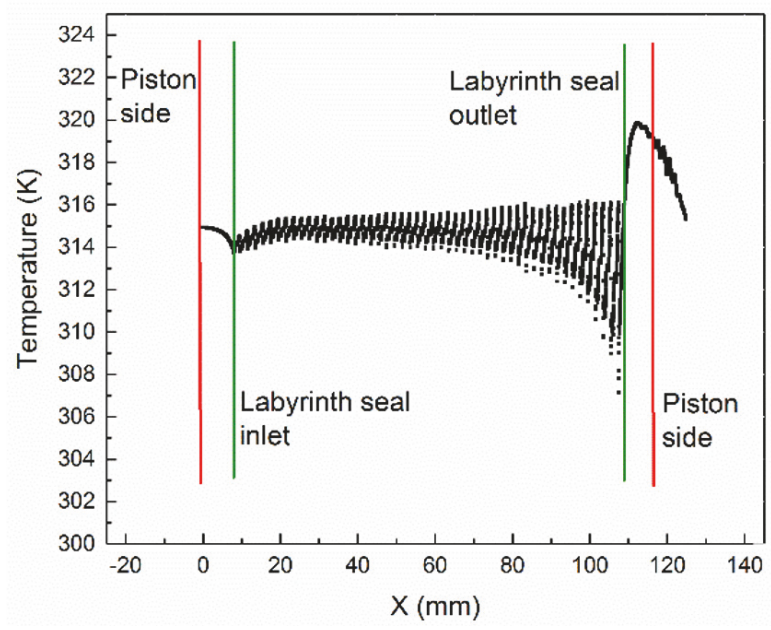

(b) Temperature along $X_{f}$

FIGURE 9: Pressure and temperature distribution.

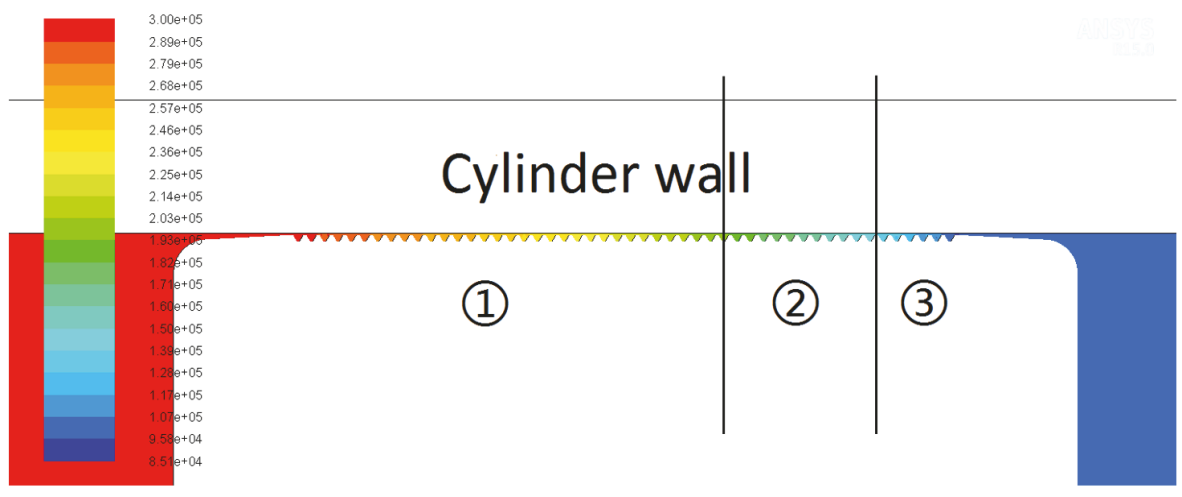

(a) Pressure drop in the labyrinth seal

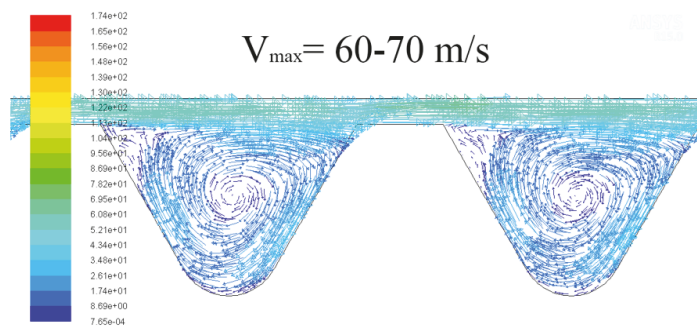

(b) Velocity field of (1)

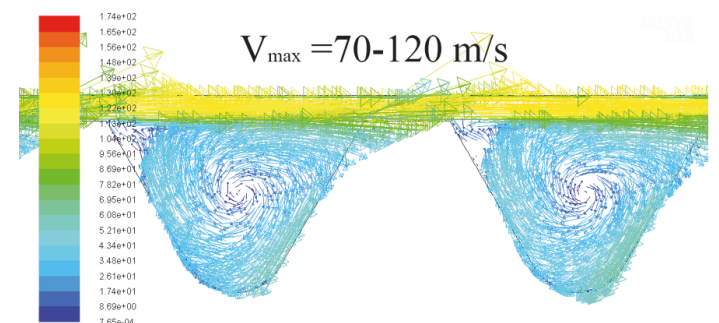

(c) Velocity field of (2)

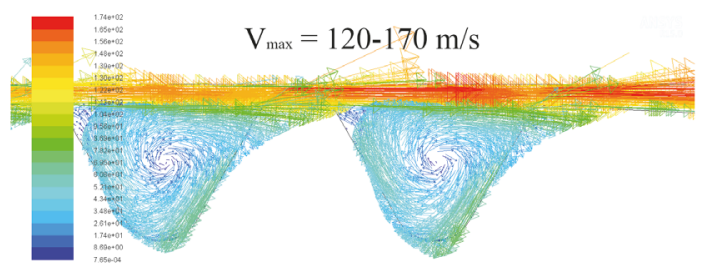

(d) Velocity field of (3)

FIGURE 10: Pressure drop and velocity fields in the labyrinth seal. 


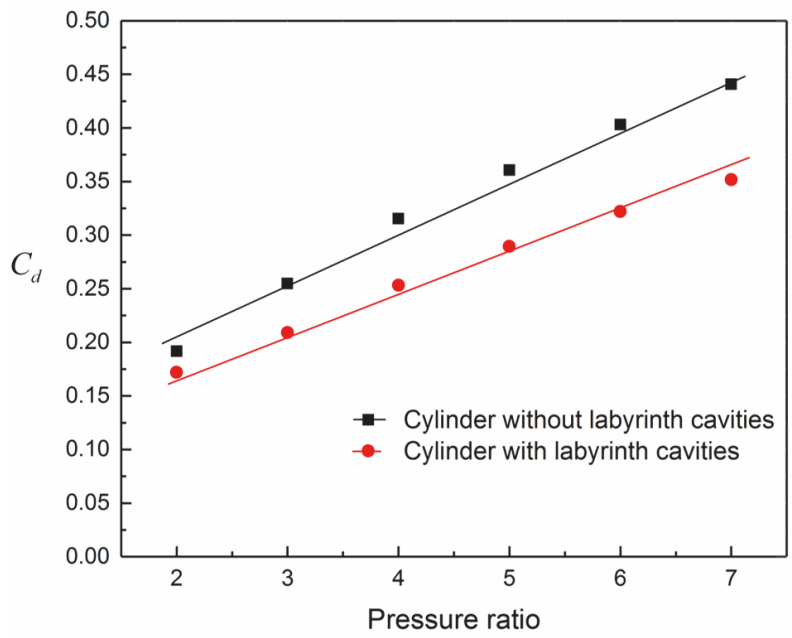

FIGURE 11: Comparison of two cylinder walls.

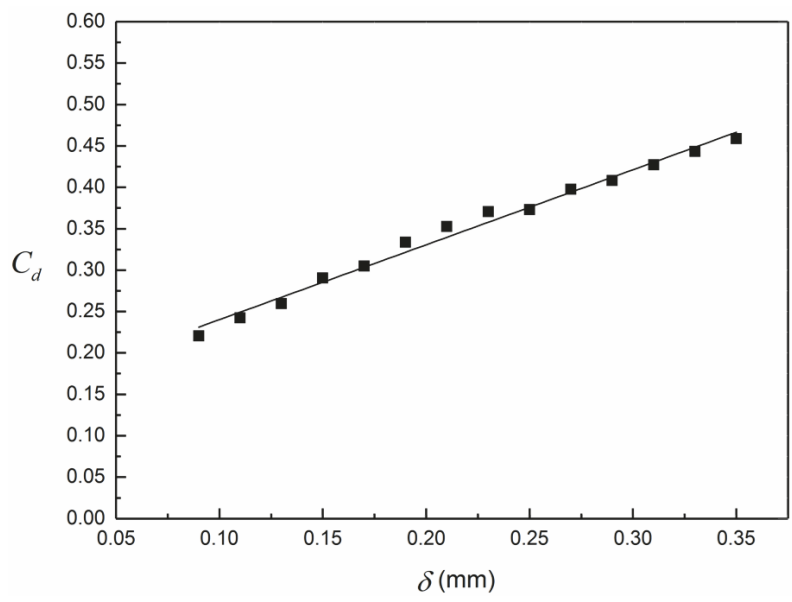

FIGURE 12: Discharge coefficient versus the labyrinth clearance.

4.4.2. Clearance. In this study, $\delta$ varied from 0.09 to 0.35 $\mathrm{mm}$. Figure 12 shows that the discharge coefficient $C_{d}$ has almost a linear growth with an increase in $\delta$. The value of $C_{d}$ increased to two times that of the original when $\delta$ increased to $0.26 \mathrm{~mm}$, indicting the significant effect of the clearance on the sealing performance. This can be attributed to the fact that when the fluid flows continuously through the labyrinth channels, a throttling occurs and the pressure drops rapidly at every tiny labyrinth clearance segment. The smaller the labyrinth clearance $\delta$ is, the stronger the throttling effect is, which results in a larger pressure drop when the fluid flows through the labyrinth clearance.

Moreover, as shown in Figure 13, the fluid on the top of the labyrinth cavity entered into the next seal directly rather than into the labyrinth cavity, where the kinetic energy will be dissipated, and thus the sealing efficiency under a wider clearance will be worse.

$\delta$ is unable to be unlimitedly narrowed in a real application owing to the assembly feasibility, and thus achieving the minimum value within the reasonable scope is of great importance for sealing. For reciprocating labyrinth piston compressors in a real application, the labyrinth clearance should be no less than $0.1 \mathrm{~mm}$, or a direct contact between the piston and cylinder wall could occur, and the inner wall of the cylinder and the piston wall will both be destroyed.

4.4.3. Number of Labyrinth Cavities. If the total length of the labyrinth clearance is constant, the tooth thickness $t$ will directly influence the number of labyrinth cavities on the piston. Let the size and shape of the labyrinth cavity remain the same, and then decrease $t$ from 150 to $0.1 \mathrm{~mm}$; the number of labyrinth cavities will then increase from 0 to 62 , with a constant uniform distribution on the piston.

Figure 14 illustrates that, with an increase in the number of labyrinth cavities to $62, C_{d}$ decreases gradually by around $36 \%$. The minimum value of $t$ was $0.1 \mathrm{~mm}$, the corresponding cavity number was 62 , and the discharge coefficient was 0.259 ; when the maximum thickness was $150 \mathrm{~mm}$, no labyrinth cavities were present on the piston, and $C_{d}$ was 0.409 . On average, one added cavity will lead to about a $0.58 \%$ decrease in $C_{d}$.

4.4.4. Cavity Width. The labyrinth cavity studied consists of two sloping edges and a circular groove at the bottom of the cavity, as shown in Figure 15. The effects of the cavity width on the leakage characteristics were investigated by changing the circle radius of the bottom groove to allow the cavity width to "zoom out" or "zoom in," as shown in Figure 16.

When the value of $w / \delta$ changed from 8 to 14 , the variation of $C_{d}$ was as shown in Figure 17. With the increase in $w / \delta, C_{d}$ increased slightly from 0.287 to 0.301 , which is only $4.88 \%$; that is, the labyrinth leakage rate increased very slightly. The velocity fields for $w / \delta=8$ and 14 are shown in Figure 18. When the depth of the labyrinth cavity was the same, the larger width led to a larger cavity, and thus the flow vortex was more drastic. Nevertheless, the larger the cavity width, the smaller the number of cavities when the whole length covered for the labyrinth seal in the piston was the same, and thus, in total, compared with the effect of the labyrinth clearance and the influence of the tooth thickness, the effect of the cavity width was insignificant. It can be concluded that changing only the size of the cavity width has a negligible influence on the labyrinth leakage.

4.5. Cavity Depth. The approach in studying the effect of the labyrinth cavity depth is similar to that of the cavity width: keep the slope angle of the cavity unchanged, simply change the circle radius of the groove, and "draw" the labyrinth cavity deeper or "compress" the cavity shallower. When $d=0.5 \sim$ $1.3 \mathrm{~mm}$, the labyrinth cavity sides were straight, but when $d<$ $0.5 \mathrm{~mm}$, the straight sides no longer existed, and therefore the corresponding radius of a small arc is taken directly as the labyrinth cavity, as shown in Figure 19(a). When $d>1.3$ $\mathrm{mm}$, a circular cavity groove could not be formed, and thus the labyrinth cavities were formed directly according to the values of $d$ and $w$, as illustrated in Figure 19(b).

Figure 20 shows that when $d / \delta$ changed incrementally from 0.6 to $2.7, C_{d}$ showed a sharp decline from 0.486 to 


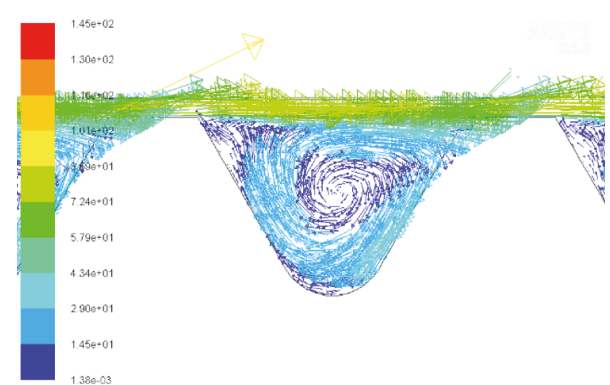

(a) $\delta=0.11 \mathrm{~mm}$

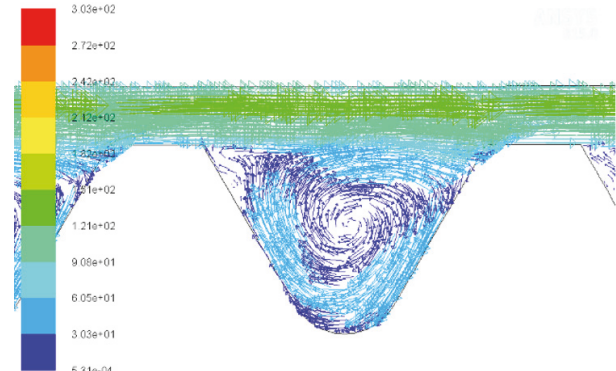

(b) $\delta=0.31 \mathrm{~mm}$

FIGURE 13: Velocity fields in different labyrinth seals $(\delta=0.11$ and $0.31 \mathrm{~mm})$.

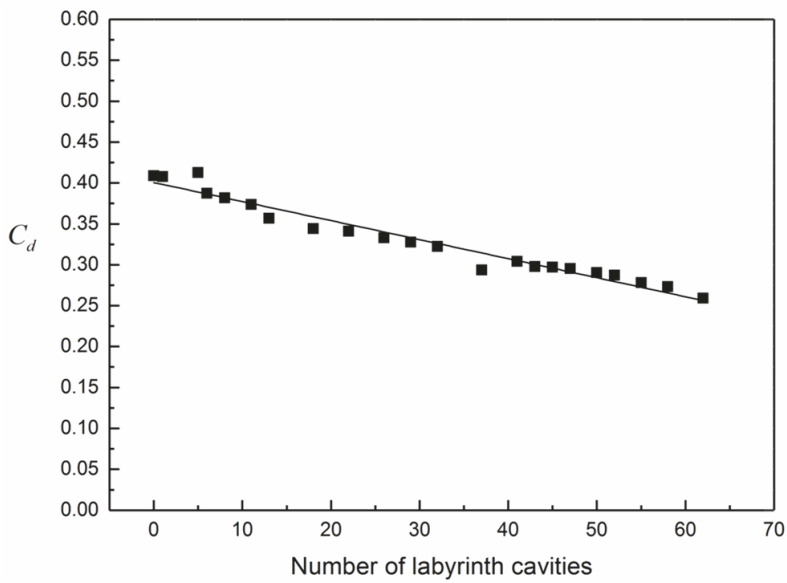

FIGURE 14: Discharge coefficient versus number of labyrinth cavities.

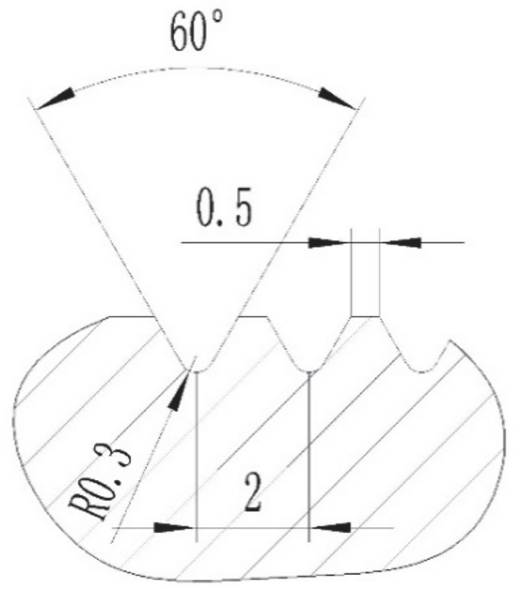

FIgURE 15: The original shape of the labyrinth cavity.

0.282 , indicating that the effect of the labyrinth seal was further enhanced more clearly with an increase in $d / \delta$. As Figure 21(a) shows, when $d / \delta=2$, the gas could not form an entire eddy within the cavity, and thus the dissipation of the kinetic energy was insufficient, and the seal efficiency was quite low.
It can also be seen that, when $d / \delta$ increased further, $C_{d}$ remained nearly unchanged at approximately 0.28 . This can be explained as a vortex forming only in the upper head of the labyrinth chamber, as shown in Figure 21(b), and thus the lower room has no contribution toward the kinetic energy dissipation. With this type of labyrinth cavity structure, when 


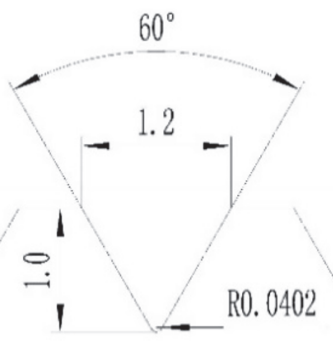

(a) $w=1.2 \mathrm{~mm}$

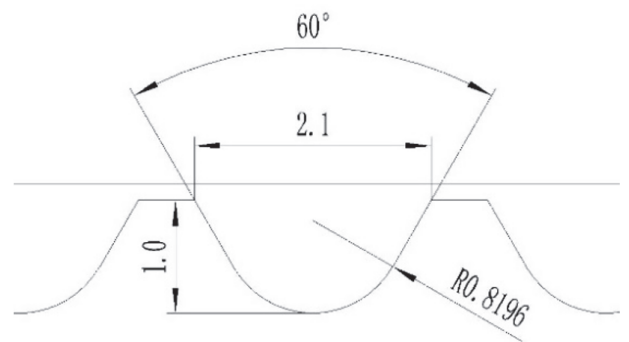

(b) $w=2.1 \mathrm{~mm}$

FIGURE 16: Change in the labyrinth cavity width.

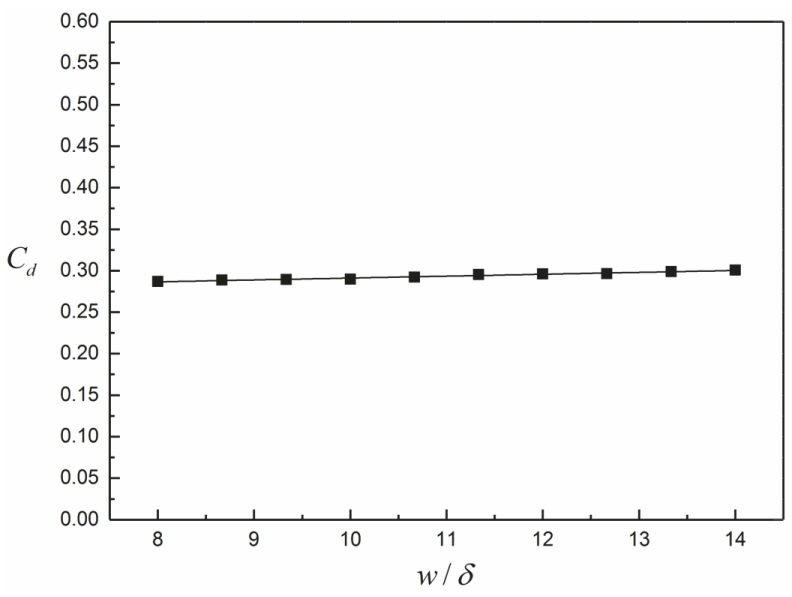

FIGURE 17: Discharge coefficient versus $w / \delta$.
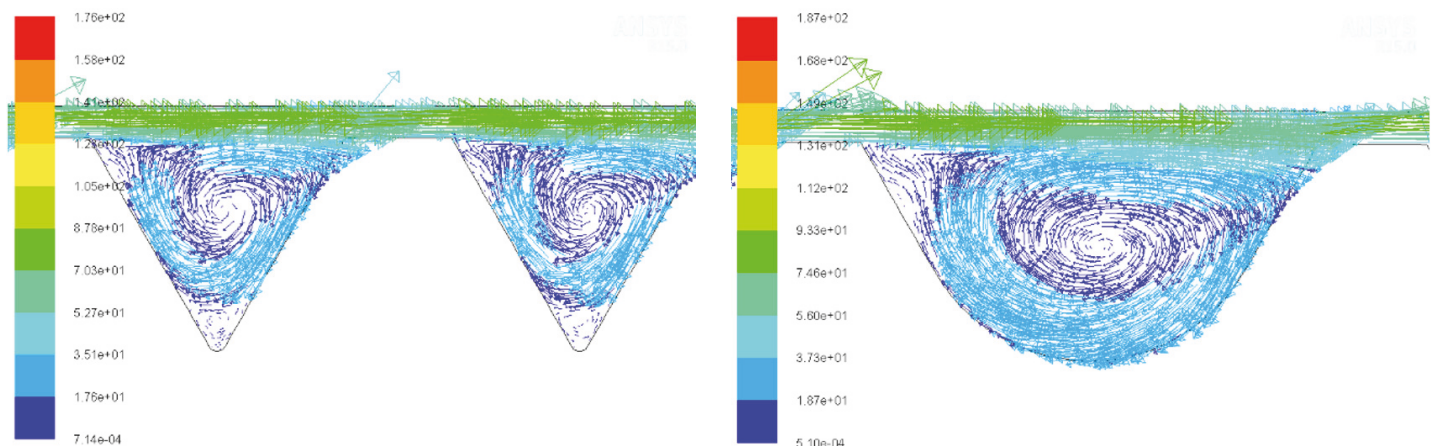

(a) $w / \delta=8.0$

(b) $w / \delta=14.0$

FIGURE 18: Velocity fields in different shapes of labyrinth cavities.

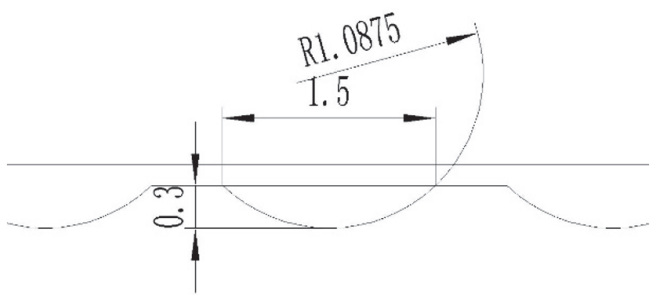

(a) $d=0.3 \mathrm{~mm}$

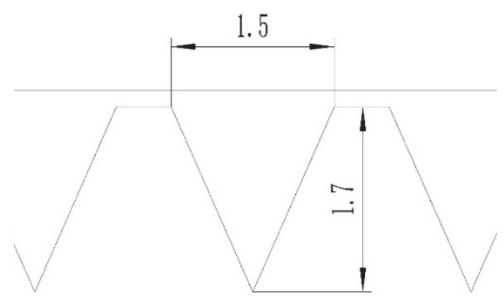

(b) $d=1.7 \mathrm{~mm}$

FIGURE 19: Change in depth of labyrinth cavity. 


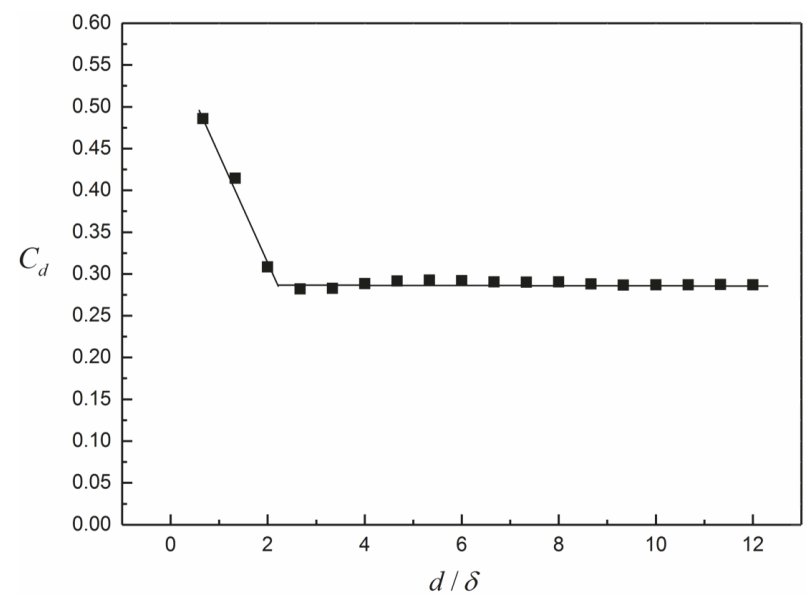

FIgURE 20: Discharge coefficient versus $d / \delta$.

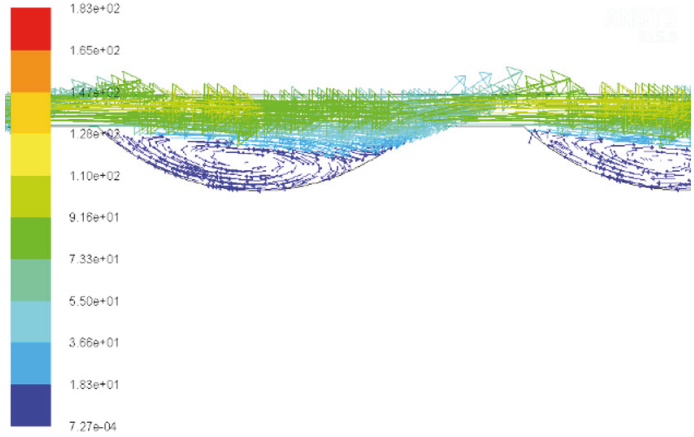

(a) $d / \delta=2.0$

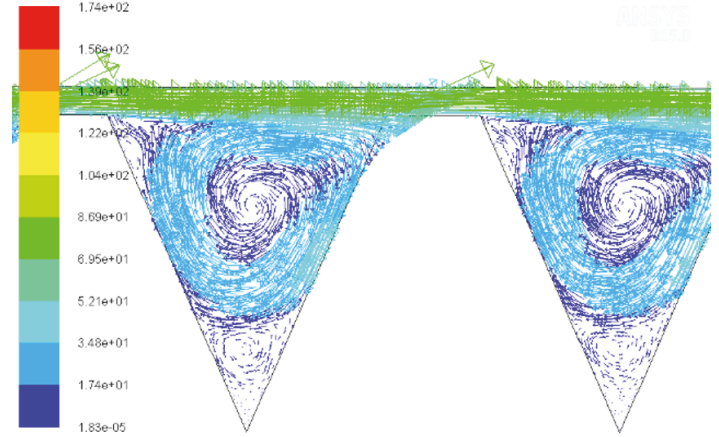

(b) $d / \delta=11.3$

FIGURE 21: Velocity fields in different shapes of labyrinth cavities.

$d / \delta$ reaches 2.7 , the sealing performance will be the highest, and no benefit can be achieved if the cavity is made deeper.

\section{Conclusions}

(1) The leakage flow rate through the labyrinth seals was shown to reduce linearly with a decrease in the clearance. When the clearance increased from 0.09 to $0.35 \mathrm{~mm}$, the discharge coefficient increased from 0.221 to 0.459 . Thus, it is important to make sure that the clearance is as small as possible when designing and assembling a labyrinth piston in practice.

(2) The value of $C_{d}$ gradually decreased by $36.7 \%$ when the number of labyrinth cavities increased from zero to 62 , and thus when the length of the labyrinth seal is constant and the shape of the labyrinth cavity is the same, the smaller the tooth thickness, the greater the number of labyrinth cavities and the higher the efficiency of the labyrinth seal that is achieved.

(3) The effect of the cavity width was not significant compared with the influence of the labyrinth clearance and the tooth thickness. Only a total change of $4.88 \%$ occurred for $C_{d}$ when $w / \delta$ increased by $75 \%$. However, because the cavity width influences the number of cavities in a piston and determines the shape of the labyrinth cavity, the value of $w$ should also be preferably controlled.

(4) For the cavity depth, when $d / \delta<2.7$, there was a sharp decline of $C_{d}$; namely, the seal efficiency clearly increased with an increase in $d$; however, if the cavity depth increased further, $C_{d}$ would barely change. Thus, $d / \delta$ should not be less than 2.7. After $d / \delta$ reaches 2.7 , further increasing this value, however, will not bring about any advantages in the sealing performance.

\section{Nomenclature}

A: Cross-sectional area of the channel

$C_{d}: \quad$ Discharge coefficient

$c_{p}: \quad$ Heat capacity

d: $\quad$ Cavity depth

$h$ : Heat transfer coefficient

$k$ : Adiabatic coefficient

$m_{\text {ideal }}$ : Ideal leakage mass flow rate

$m_{\text {real }}$ : Real leakage mass flow rate

$\rho: \quad$ Density

$q$ : Heat flux

$\mu$ : $\quad$ Dynamic viscosity

$R: \quad$ Molar gas constant 


\author{
S: Mass force \\ $\tau$ : Stress tensor \\ $t$ : Labyrinth tooth thickness \\ T: Temperature \\ $\delta$ : Labyrinth clearance \\ $v$ : Velocity \\ $w$ : Cavity width \\ $\phi$ : Variables in computational domain.
}

\section{Data Availability}

All data are fully provided in the Results Discussion.

\section{Conflicts of Interest}

The authors declare no potential conflicts of interest with respect to the research, authorship, and/or publication of this article.

\section{Acknowledgments}

This work was supported by the Natural Science Foundation of Shaanxi Province, China [Research Project 2016JQ5114].

\section{References}

[1] Engineering Science Data Unit, "Labyrinth seal flow," HIS ESDU ESDU 09004, Denver, CO, USA, 2009.

[2] H. M. Martin, "Labyrinth packing," Engineering, vol. 1, pp. 3536, 1908.

[3] A. Egli, "The leakage of steam through labyrinth seals," Journal of Heat Transfer, ASME, vol. 57, pp. 115-122, 1935.

[4] B. Hodkinson, "Estimation of the Leakage through a Labyrinth Gland," Proceedings of the Institution of Mechanical Engineers, vol. 141, no. 1, pp. 283-288, 1939.

[5] L. M. Milne-Thomson, Theoretical aerodynamics, Macmillan and Company, Ltd, London, UK, 2nd edition, 1949.

[6] G. Vermes, "A fluid mechanics approach to the labyrinth seal leakage problem," Journal of Engineering for Gas Turbines and Power, vol. 83, no. 2, pp. 161-169, 1961.

[7] S. Wittig, U. Schelling, S. Kim, and K. Jacobsen, "Numerical Predictions and Measurements of Discharge Coefficients in Labyrinth Seals," in Proceedings of the ASME 1987 International Gas Turbine Conference and Exhibition, p. V001T01A064, Anaheim, California, USA.

[8] J. A. Demko, G. L. Morrison, and D. L. Rhode, "The prediction and measurement of incompressible flow in a labyrinth seal," Journal of Engineering for Gas Turbines and Power, vol. 111, no. 4, pp. 697-702, 1989.

[9] H. L. Stocker, "Determining and improving labyrinth seal performance in current and advanced high performance gas turbines," in Proceedings of the AGARD conference Proceedings, pp. 237-13, 1978.

[10] D. Sun, S. Wang, Z. Xiao, J. Meng, X. Wang, and T. Zheng, "Measurement versus predictions of rotordynamic coefficients of seal with swirl brakes," Mechanism and Machine Theory, vol. 94, pp. 188-199, 2015.

[11] L. S. Andrés and A. Anderson, "An All-Metal Compliant Seal Versus a Labyrinth Seal: A Comparison of Gas Leakage at
High Temperatures," Journal of Engineering for Gas Turbines and Power, vol. 137, no. 5, 2015.

[12] X. Li, J. Yang, and W. Xu, "Research and comparison on the leakage and fluid force between the axial and the radial labyrinth seal," Journal of Mechanical Science and Technology, vol. 29, no. 11, pp. 4611-4620, 2015.

[13] M. Flouros, P. Hendrick, B. Outirba, F. Cottier, and S. Proestler, "Thermal and flow phenomena associated with the behavior of brush seals in aero engine bearing chambers," Journal of Engineering for Gas Turbines and Power, vol. 137, no. 9, 2015.

[14] Z. Li, J. Li, and Z. Feng, "Numerical comparison of rotordynamic characteristics for a fully partitioned pocket damper seal and a labyrinth seal with high positive and negative inlet preswirl," Journal of Engineering for Gas Turbines and Power, vol. 138, no. 4, 2016.

[15] W. Wang, Y. Liu, P. Jiang, and H. Chen, "Numerical analysis of leakage flow through two labyrinth seals," Journal of Hydrodynamics, vol. 19, no. 1, pp. 107-112, 2007.

[16] S. Suryanarayanan and G. L. Morrison, "Analysis of Flow Parameters Influencing Carry-Over Coefficient of Labyrinth Seals," in Proceedings of the ASME Turbo Expo 2009: Power for Land, Sea, and Air, pp. 1137-1145, Orlando, Florida, USA.

[17] S. Suryanarayanan and G. L. Morrison, "Effect of tooth height, tooth width and shaft diameter on carry-over coefficient of labyrinth seals," in Proceedings of the 2009 ASME Turbo Expo, pp. 1147-1152, USA, June 2009.

[18] S. Suryanarayanan and G. L. Morrison, "Labyrinth seal discharge coefficient for rectangular cavities," in Proceedings of the ASME 2009 Fluids Engineering Division Summer Meeting, Colorado, USA, 2009.

[19] T. S. Kim and K. S. Cha, "Comparative analysis of the influence of labyrinth seal configuration on leakage behavior," Journal of Mechanical Science and Technology, vol. 23, no. 10, pp. 28302838, 2009.

[20] L. Bozzi, E. D’Angelo, B. Facchini, M. Micio, and R. Da Soghe, "Experimental investigation on leakage losses and heat transfer in a non conventional labyrinth seal," in Proceedings of the ASME 2011 Turbo Expo: Turbine Technical Conference and Exposition, GT2011, pp. 955-965, Canada, June 2011.

[21] A. O. Pugachev, Y. A. Ravikovich, and L. A. Savin, "Flow structure in a short chamber of a labyrinth seal with a backwardfacing step," Computers \& Fluids, vol. 114, pp. 39-47, 2015.

[22] A. O. Pugachev, M. Gaszner, C. Georgakis, and P. Cooper, "Segmentation Effects on Brush Seal Leakage and Rotordynamic Coefficients," Journal of Engineering for Gas Turbines and Power, vol. 138, no. 3, 2016.

[23] H. Yuan, S. Pidaparti, M. Wolf, J. Edlebeck, and M. Anderson, "Numerical modeling of supercritical carbon dioxide flow in see-through labyrinth seals," Nuclear Engineering and Design, vol. 293, pp. 436-446, 2015.

[24] Q. Meng and G. Xia, "Analysis design and test of reducing leakage rate of labyrinth seal," Electro-optic technology application, vol. 29, no. 3, pp. 81-84, 2014.

[25] T.-H. Shih, W. W. Liou, A. Shabbir, Z. Yang, and J. Zhu, "A new $k$ - $\epsilon$ eddy viscosity model for high reynolds number turbulent flows," Computers \& Fluids, vol. 24, no. 3, pp. 227-238, 1995.

[26] S. M. Panicker, eakage prediction of labyrinth seals having advanced cavity shapes. M. S. thesis, Texas A\&M University, College Station, TX, USA, 2010.

[27] J. Denecke, J. Färber, K. Dullenkopf, and H. Bauer, "Interdependence of Discharge Behavior, Swirl Development and Total 
Temperature Increase in Rotating Labyrinth Seals," in Proceedings of the ASME Turbo Expo 2008: Power for Land, Sea, and Air, pp. 1717-1724, Berlin, Germany.

[28] Y. Kang, T. S. Kim, S. Y. Kang, and H. K. Moon, "Aerodynamic Performance of Stepped Labyrinth Seals for Gas Turbine Applications," in Proceedings of the ASME Turbo Expo 2010: Power for Land, Sea, and Air, pp. 1191-1199, Glasgow, UK.

[29] K. C. Nayak and P. Dutta, "Numerical investigations for leakage and windage heating in straight-through labyrinth seals," Journal of Engineering for Gas Turbines and Power, vol. 138, no. 1, 2016. 


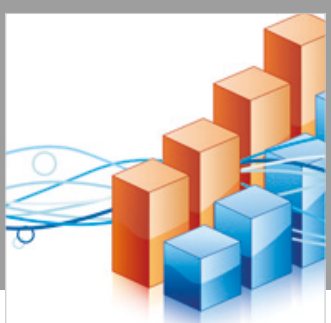

Advances in

Operations Research

\section{-n-m}
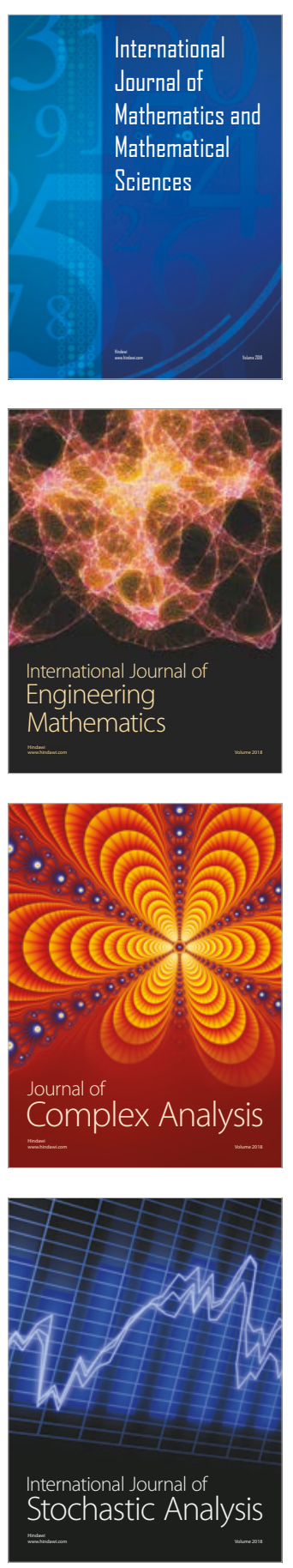
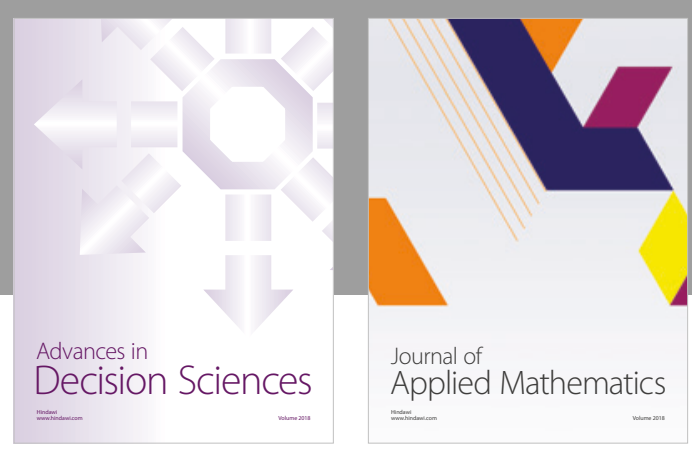

Journal of

Applied Mathematics
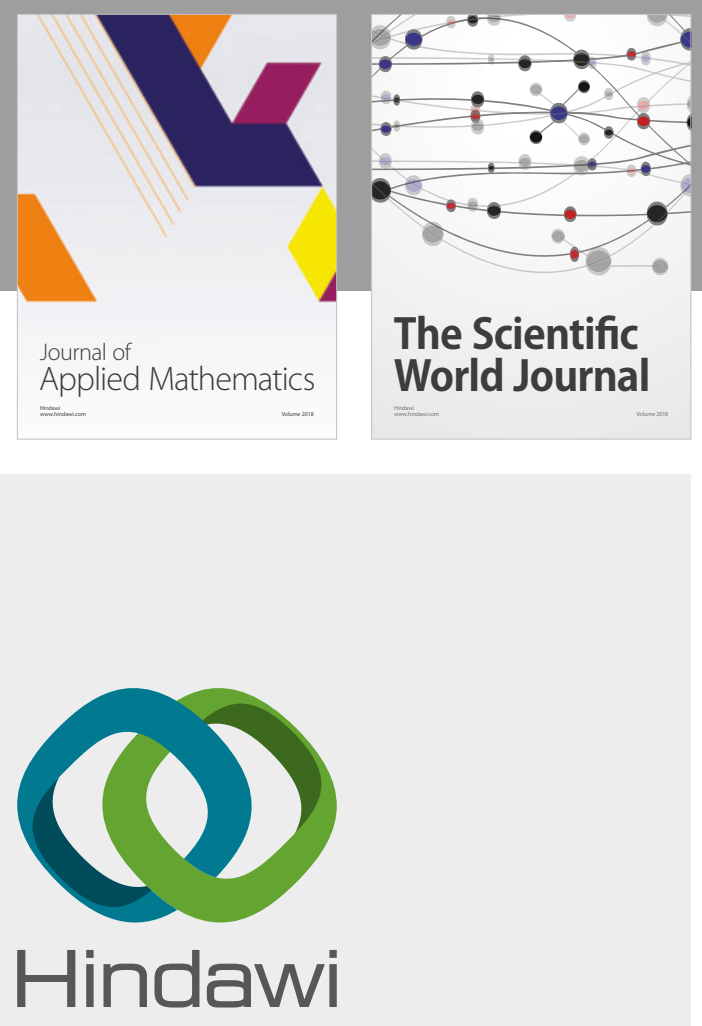

Submit your manuscripts at

www.hindawi.com

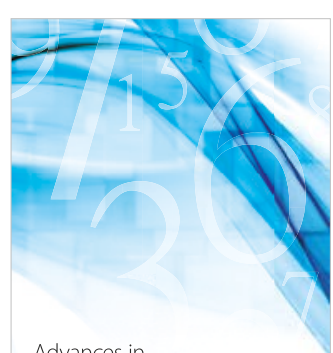

Advances in
Numerical Analysis
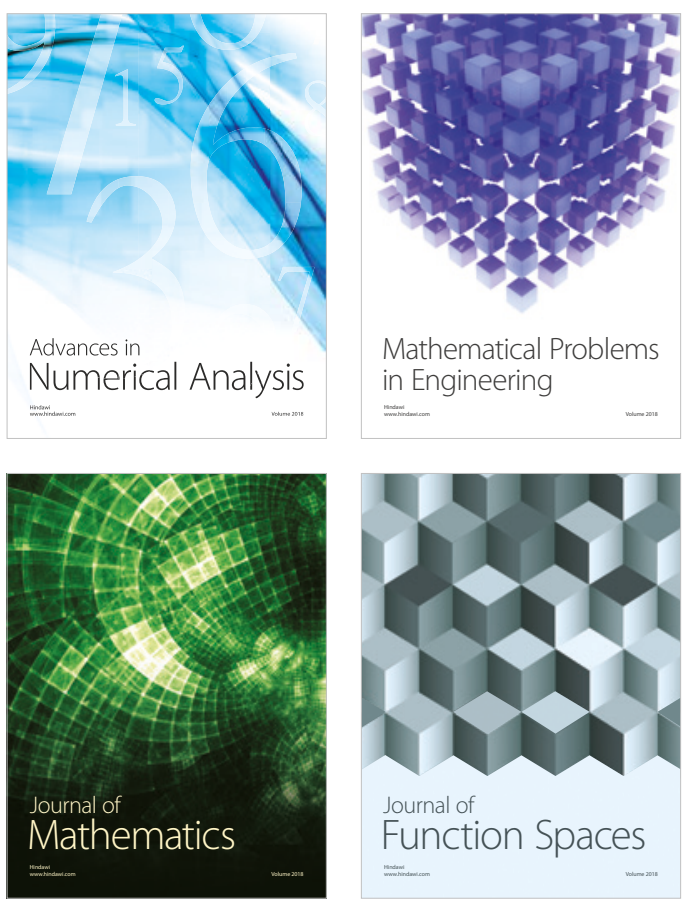

Mathematical Problems in Engineering

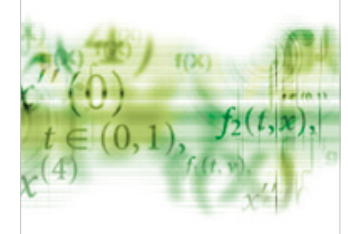

International Journal of

Differential Equations

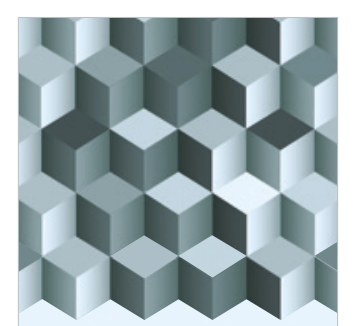

Journal of

Function Spaces

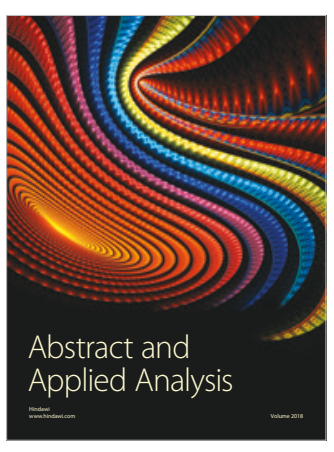

The Scientific

World Journal

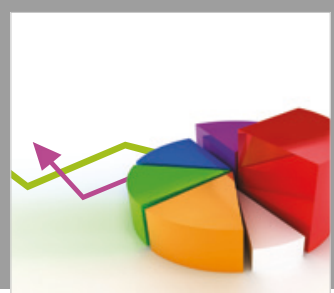

Journal of

Probability and Statistics
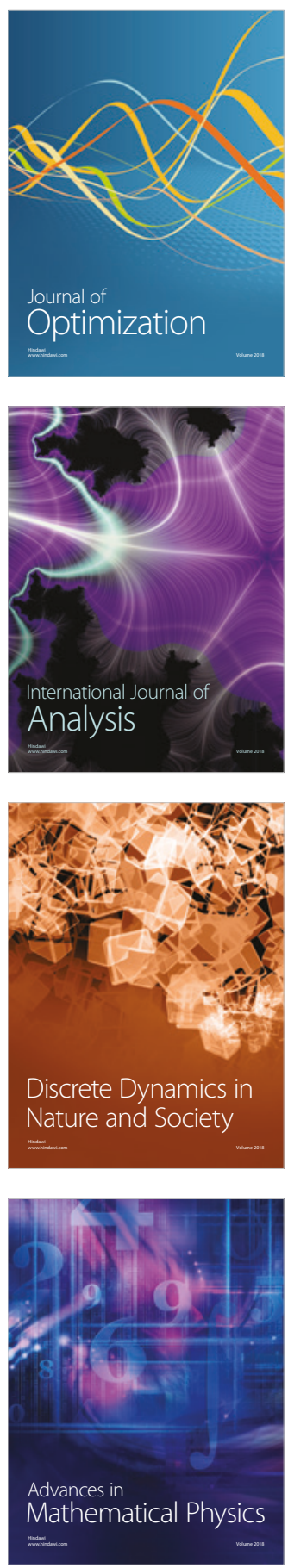